\title{
COMPOSITE COMPLEX TECHNICAL SOLUTIONS AS A NECESSARY BASIC ACTIVATING FOUNDATION FOR CREATING COMBINED SOFTWARE PRODUCTS-EQUIVALENTS OF COMPLEX SYSTEM INTEGRATIVE INVENTIONS
}

\author{
Konstantin Piliugin \\ Leading Front-end Engineer of ClickUp Inc. \\ Russian Federation, Voronezh
}

Due to the introduction into everyday practice of various kinds of combined software products, usually including many interrelated elements not interconnected in early design versions, there was a real need to create composite and complex technical solutions where all elements previously not interconnected form a composite complex technical solution.

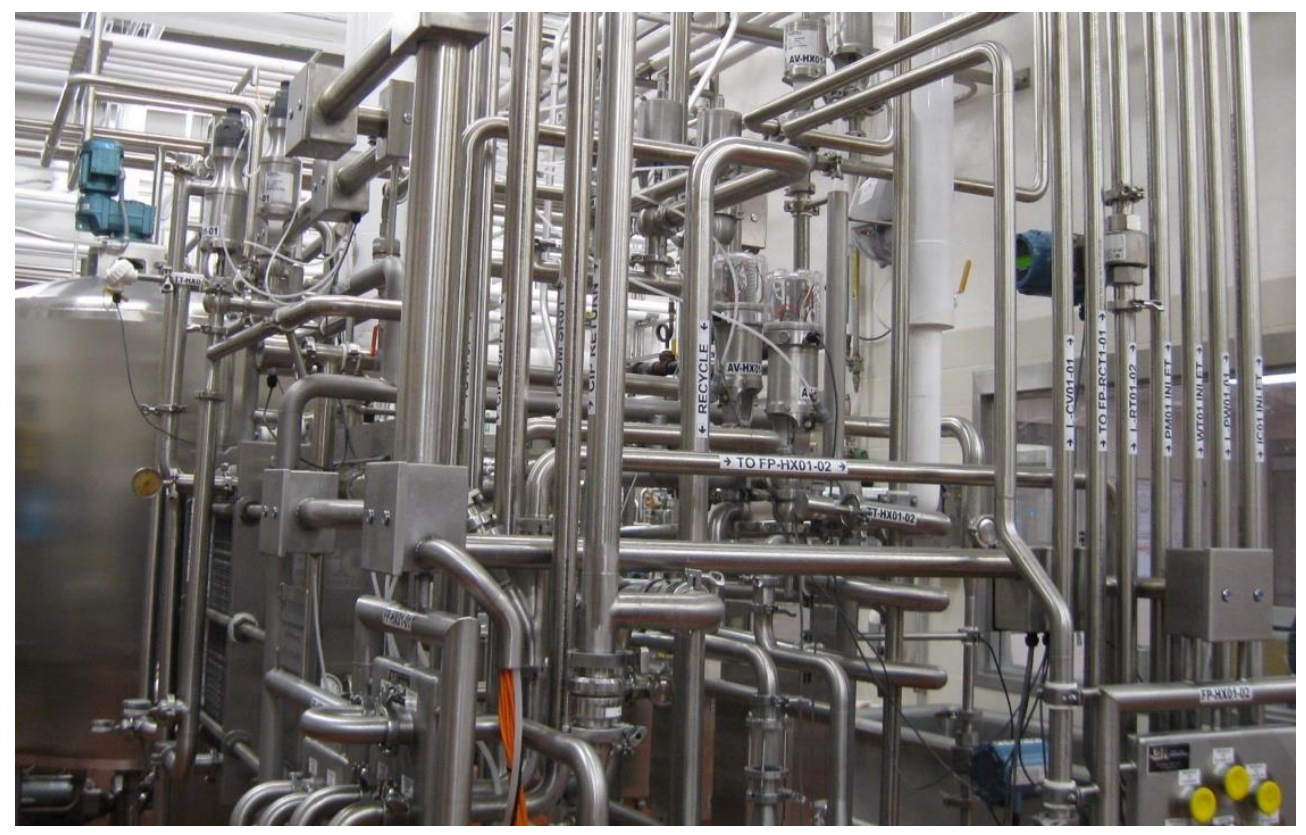

Figure 1. An example of a composite complex technical solution that includes combined software products, aligned with complex system integrative inventions in the field of environmentally friendly and fully automated dairy production 
Almost complete transition of the economies of developed countries to innovative methods of planning and management dictates new criteria for the qualification of technical solutions used.

Gradually, any kind of production in such conditions, in order to maintain the necessary level of competitiveness - shall transform and optimize its technological and commercial assets to achieve the level of so-called smart production.

But it does not make much sense to create production, for production, without a serious well thought out strategic framework, including in terms of the most effective information tool for attracting partners and customers, such as web sites with a dynamic information system.

Today, more and more attention is paid to this process as part of smart production, including parallel creation of appropriate software techniques and tools, including, of course, three-dimensional graphics elements that carry more and more information containing data of innovative tools, equipment and materials used.

Taking into account the fact that, for example, in the USA, new patent legislation has already been adopted and introduced, it makes sense to dwell in greater detail on the feasibility of including in these information materials on the latest developments used in the advertised products and which can be qualified for compliance with the four basic signs of invention. This would be, first of all, the criterion of non-obviousness of the invented technical solution.

Of course, in such a situations, for commercial reasons, it is important to preserve all possible levels of protection of the rights to the original intellectual property, even in cases when reliable protection has not yet been created and the product is already being delivered. 
What kind of information and in what form can bring the level of information able to form a positive perception of the new product and all information related thereto, to concerned parties?

First of all, such information may include data on fundamentally new materials used in a new innovative product and their combinations with traditional materials that are quite well known in the market.

Of course, the disclosure of all details and features of the application must be approached with a known and sufficient degree of caution, since violation of the criteria of absolute novelty may close the way to patenting a product or its components.

A huge role in ensuring the proper technical level of new products and their consumer qualities is played by the presence of composite materials therein, especially carboncarbon compositions.

Innovative composite materials are usually combined with innovative manufacturing technology, also in terms of smart production, and the most valuable quality of such smart production is the capability to monitor quality in real-time at all stages of the production process.

Finally, the nature of information presentation, including on websites, is influenced and affected by new and modernized consumer standards as well as new environmental standards and their perception in each of the specific areas of the consumer market.

\section{Availability of fundamentally new materials}

Fundamentally new materials today can come in a wide variety of types and purposes and can be used in innovative products for a variety of reasons.

One of the most important aspects of the search for and use of fundamentally new structural materials is the need to use processing equipment with digital software control to manufacture parts and elements of a new product. 
This equipment has a place for new structural tool materials and alloys, and especially cutting materials of increased hardness and durability.

Recently, metal-ceramic materials and their variants have become especially popular in this area.

The use of such materials significantly increases the reliability and accuracy of metal-cutting tools, as well as equipment performance.

With the emergence of 3D-injection molding machines, various plastics and their ceramic equivalents are increasingly required for construction materials.

Variance can be very broad and we can talk about a variety of final products, significantly differing in many technical characteristics, including mass and weight characteristics, as well as practical areas of application.

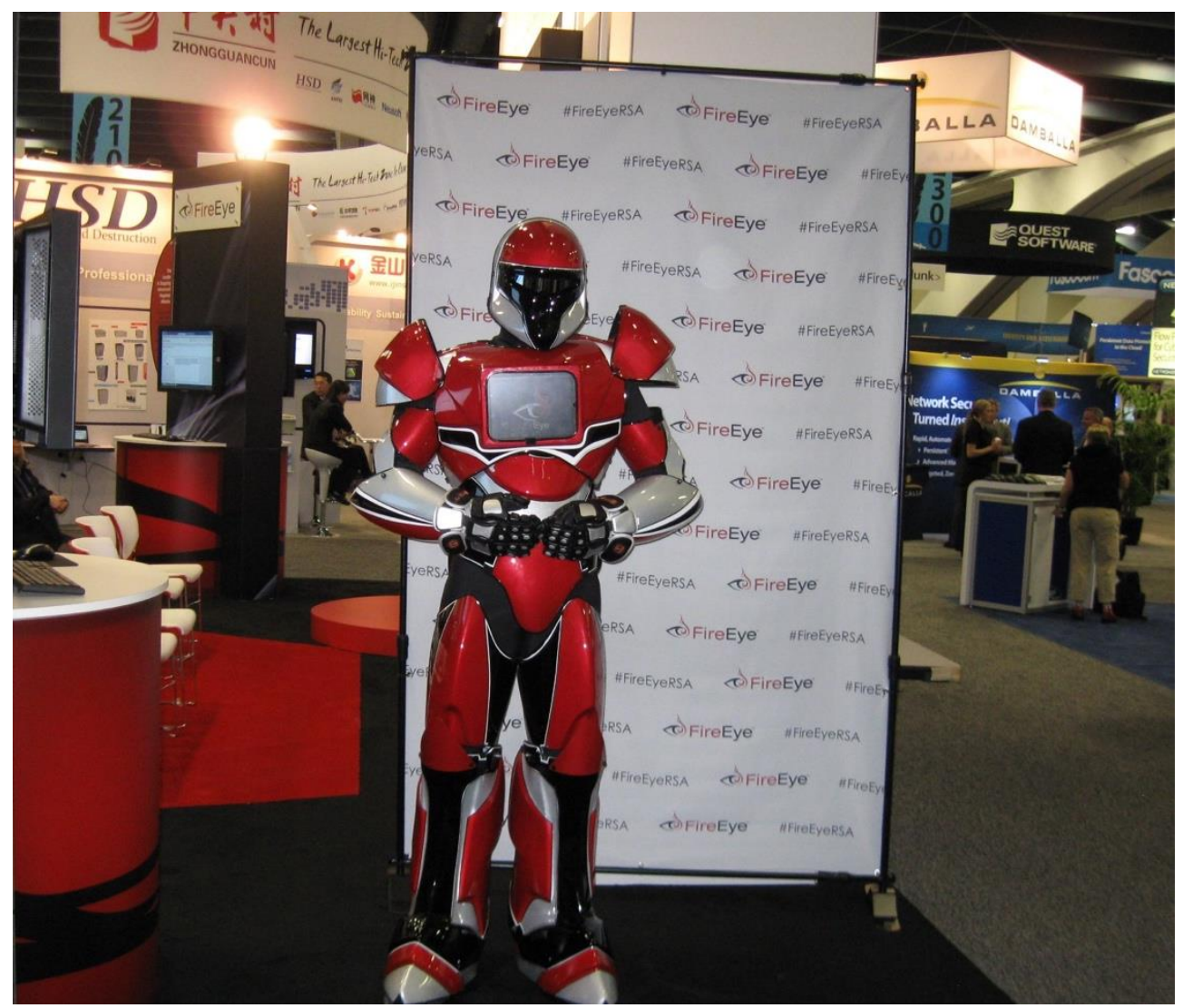


Figure 2. An example of the use of fundamentally new materials in fire protection demonstrated at the international exhibition of protective technologies and materials;

This type of information does not reveal production, technological, constructive and operational secrets and at the same time allows us to bring all the winning parameters and capabilities of new products to potential customers

As is known from the patent legislation, just the replacement of the used construction material with a new one, or even a fundamentally new one, is not an invention.

On the other hand, such a simple replacement is not always possible at all. Complete replacement of the material requires other additional technical solutions, including both schematic and component ones.

As a rule, in combination with the fundamental solutions on replacement of the material and on changes in the product properties and parameters arising from changes in the properties of the new material, a comprehensive technical solution arises, and its main distinguishing features form compliance of the invention with criteria, including on the basis of non-obviousness of the technical solution for the specialist of the average qualification in this area.

Most often, if a new product is developed, manufactured and tested in one working group or in a start-up team, patent search and qualification of a technical solution for compliance with signs can be carried out only after all production test cycles and final qualification tests of the actual technical feature of a new product.

If a new product includes a controlling and monitoring processor or a group of processors, then the nature of the search by the proposed invention expands, and the search pattern changes, just like the invention structure.

The invention scheme takes the following form - design, system, program, and method. 
Thus, novelty of the distinctive features of an innovative product consists of several mutually dependent attributes relating to various elements of the working characteristic.

Particular interest in this process of integrative identification of new technical solutions is paid to the use of fundamentally new structural materials and technological solutions, emulsions and aerosols.

The method of presenting the information of these novelties to potential users of these products today is largely determined by the level of information load of the web product of the developer and manufacturer of this product.

This is because in principle, in accordance with the patent laws of the United States and other countries with innovative economies, simply replacing a material, even with one which is the newest and most advanced, is not an invention. Correct presentation of technological and constructive gains on the website without violating the product novelty, allows the maintaining of the principle of novelty and at the same time brings all the information required for decision-making to the attention of parties potentially interested in an innovative product.

The line between fundamentally new materials and composite materials can be quite narrow, but it still makes sense to allocate a separate place for composite materials.

\section{Presence of composite materials}




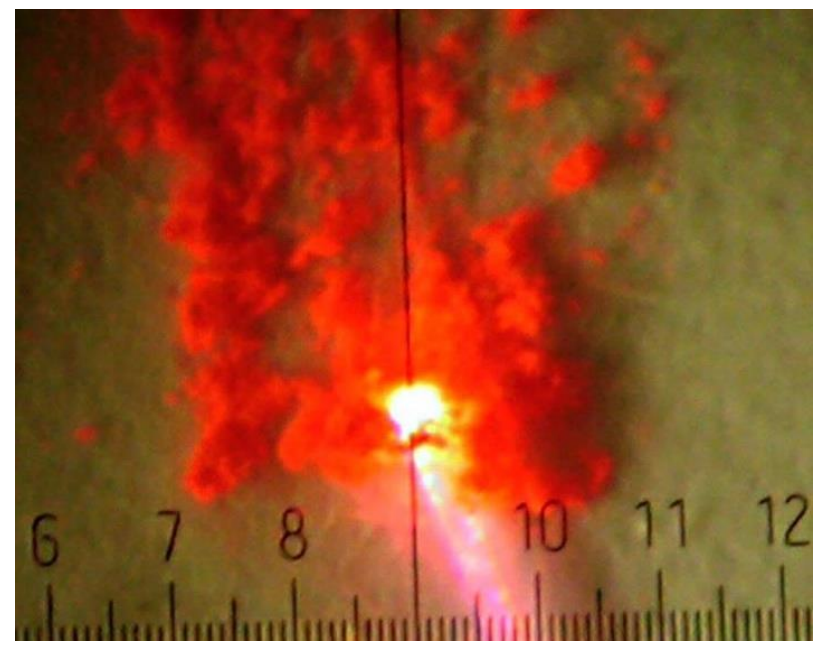

Figure 3A. An example of the use of innovative composite materials - luminophors in laser technology and fiber optics;

Red luminophor

Luminophors shown in Figure 3A and Figure 3B are used in disposable surgical laser instruments.

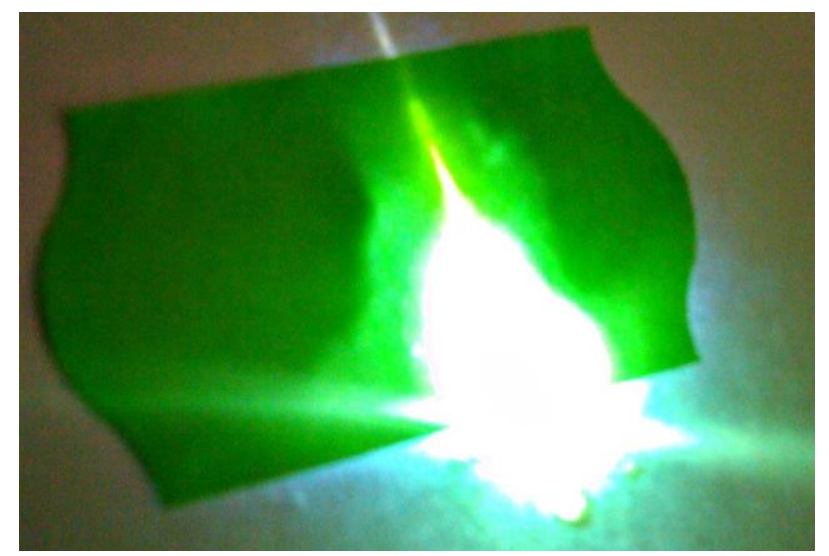

Figure 3B. An example of the use of innovative composite materials - luminophors in laser technology and fiber optics;

Green luminophor

The use of composite materials fundamentally changes even the principles of industrial design - if earlier, when selecting construction materials, the choices made were 
guided by the materials' properties and parameters, strength, elasticity, chemical resistance, durability, specific gravity, electrical resistance, etc. Now when using composite materials, it becomes possible to control the properties and quality of composites, changing the composition of their ingredients and their interrelations.

This is especially noticeable when using carbon-carbon composites. Such composites are produced by multilayer pyrolysis in a vacuum on a substrate of viscose fabric.

The number of pyrolysis layers can be changed depending on the need and because of this, it is possible to define the properties of the material in advance. Thus, the same equipment, the so-called tunnel vacuum furnaces, can produce both flexible and rigid electrodes for electrochemical cells and flexible permeable contacts for electrochemical reactors.

All of the above shows that, on the basis of the same technological methods, it is possible to make various innovative products practically on the same technological equipment.

One of the tasks of the combined software products is targeted coverage. For example, on the website, this could be targeted coverage of all aspects of innovative applications of composite materials and targeted adjustment of output parameters of final products by adjusting the parameters of composites used in their manufacture.

\section{New directions in the technology of manufacturing elements of products and innovative items, including in conditions of smart production}

Technological production lines of modern enterprises are complex and expensive systems and complexes, and each change in production conditions and requirements introduces significant changes and adjustments to the lines. 


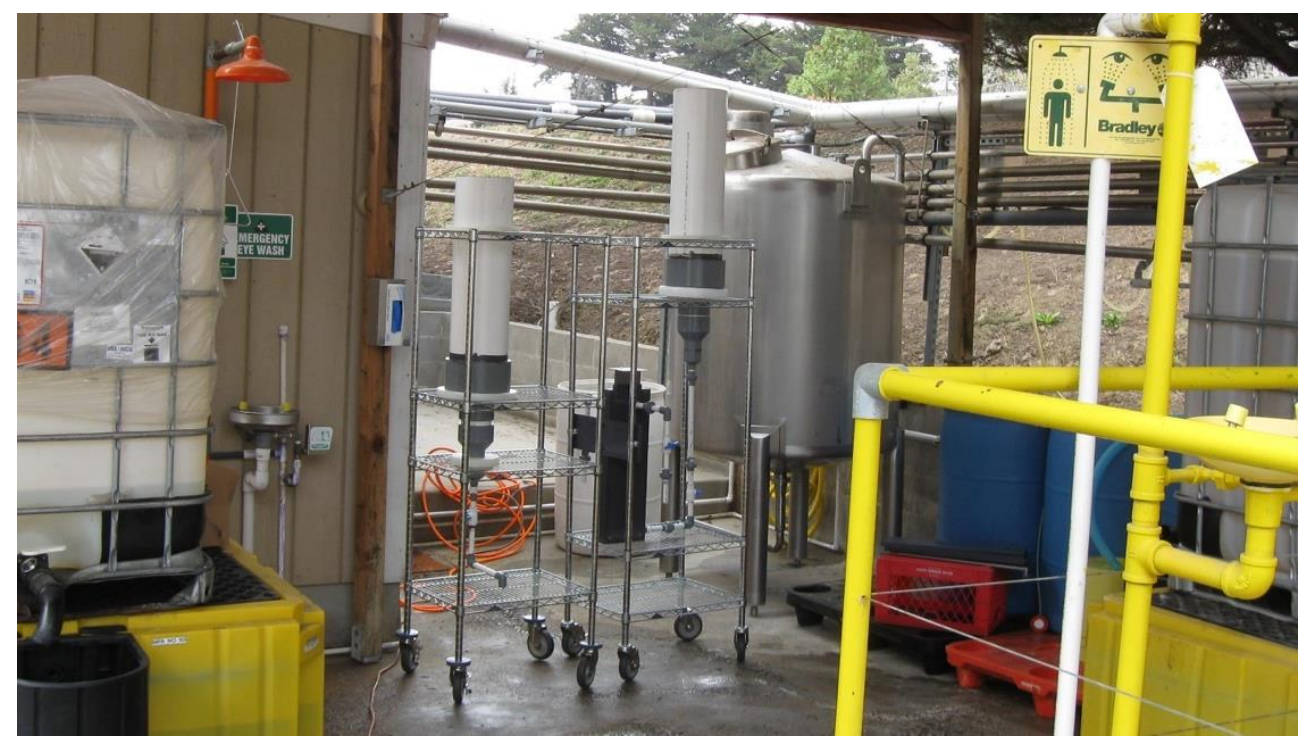

Figure 4. An example of experimental embedding of an innovative system for regeneration of process water without the use of chemical reagents in a typical modern food production facility;

an electrochemical reactor which is an integrative invention, is used in the system as the main instrument of electrochemical impact on polluted water

In this situation, the most important thing is not to change the main equipment, and, if possible, to modernize it, embedding new elements into the existing technological line.

In many ways, this possibility of substantial modernization with minimal changes and, accordingly, with minimal costs, determines the reality of the introduction of new products and technologies at existing enterprises.

New consumer standards 


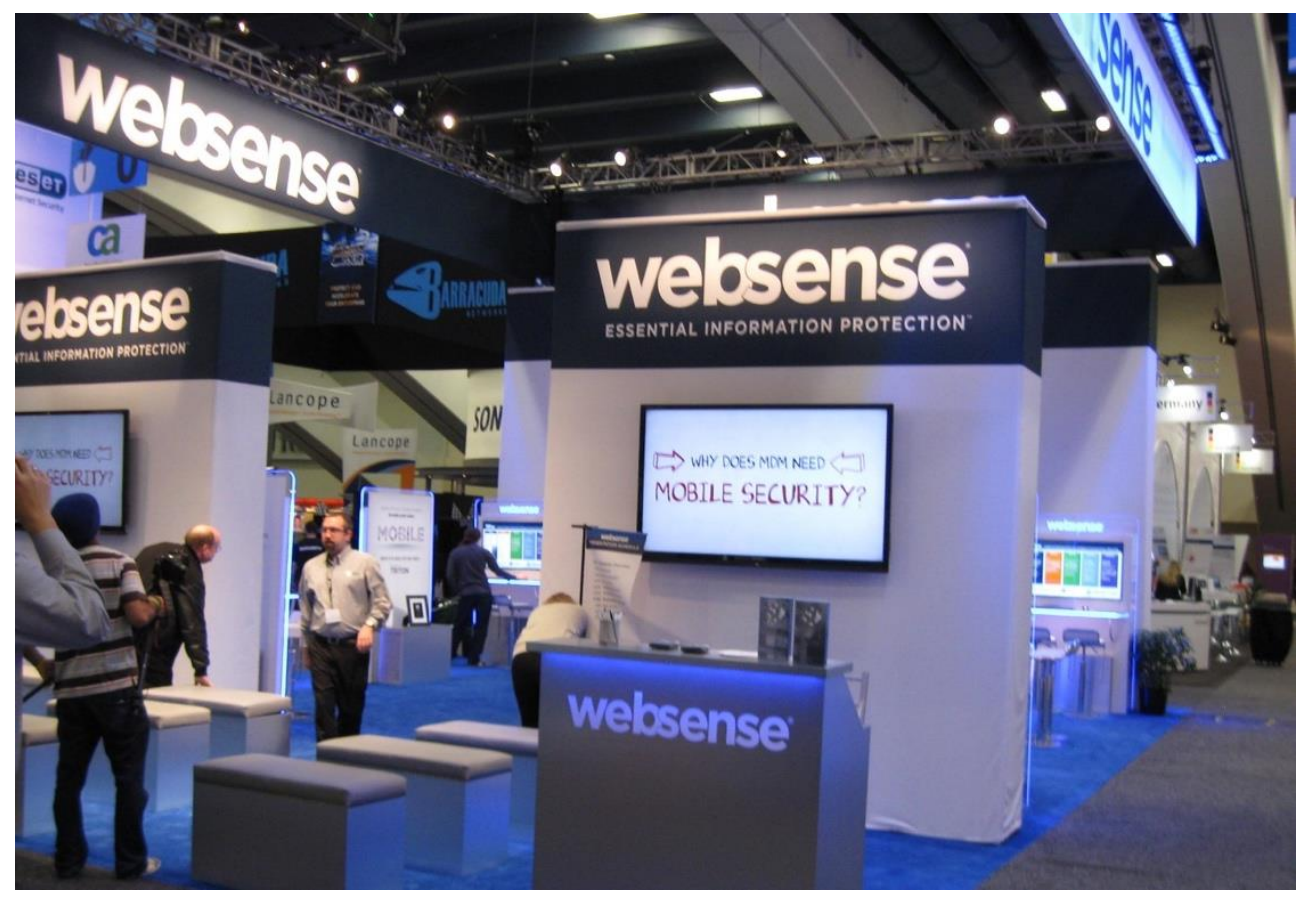

Figure 5. An example of the developers' response to the increased demands of consumers for the level of cyber security, including for web resources

Composite commercial solutions in an innovative economy require an appropriate approach from developers at all levels and especially developers of combined software products, including website developers.

Consumers today focus on ensuring full protection of information, with maximum openness and full clarity of the material presented.

In turn, developers of relevant software products are also interested in the possibility of maximum possible protection of their developments while at the same time maximizing the opportunity to provide users with the fullest informational picture possible.

\section{New environmental standards}




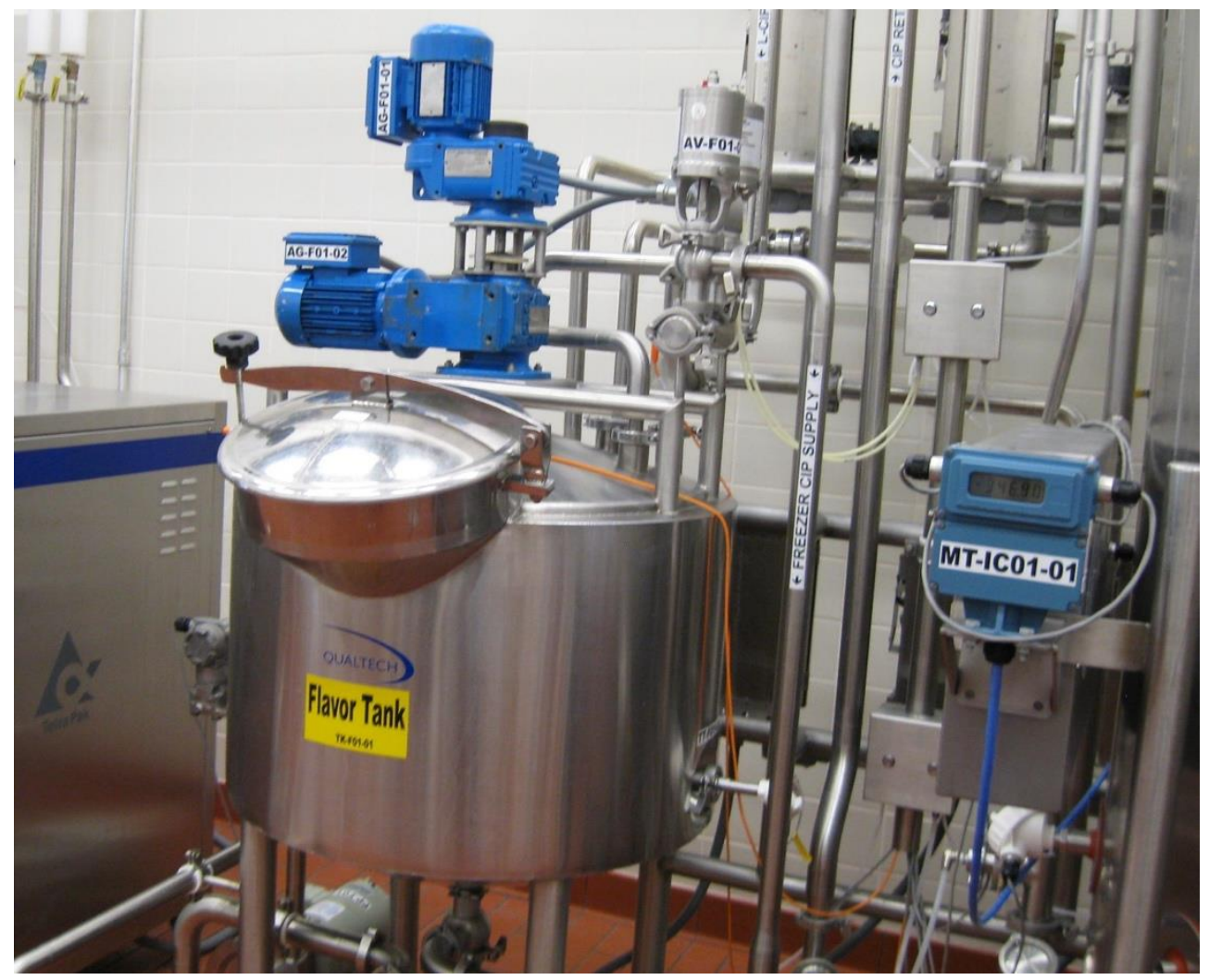

Figure 6. An example of a highly pure and environmentally friendly technological module in an enterprise producing high-quality food; The readers are invited to assess the degree of purity and compliance against the most stringent requirements of environmental standards

Despite the fact that controversial elements are constantly found in the interpretation of requirements and limitations of environmental standards, the control technique itself is constantly being improved. Likewise, the software for consistency and accuracy of control is also being optimized and modified in parallel with the development of new measurement equipment and technology. 


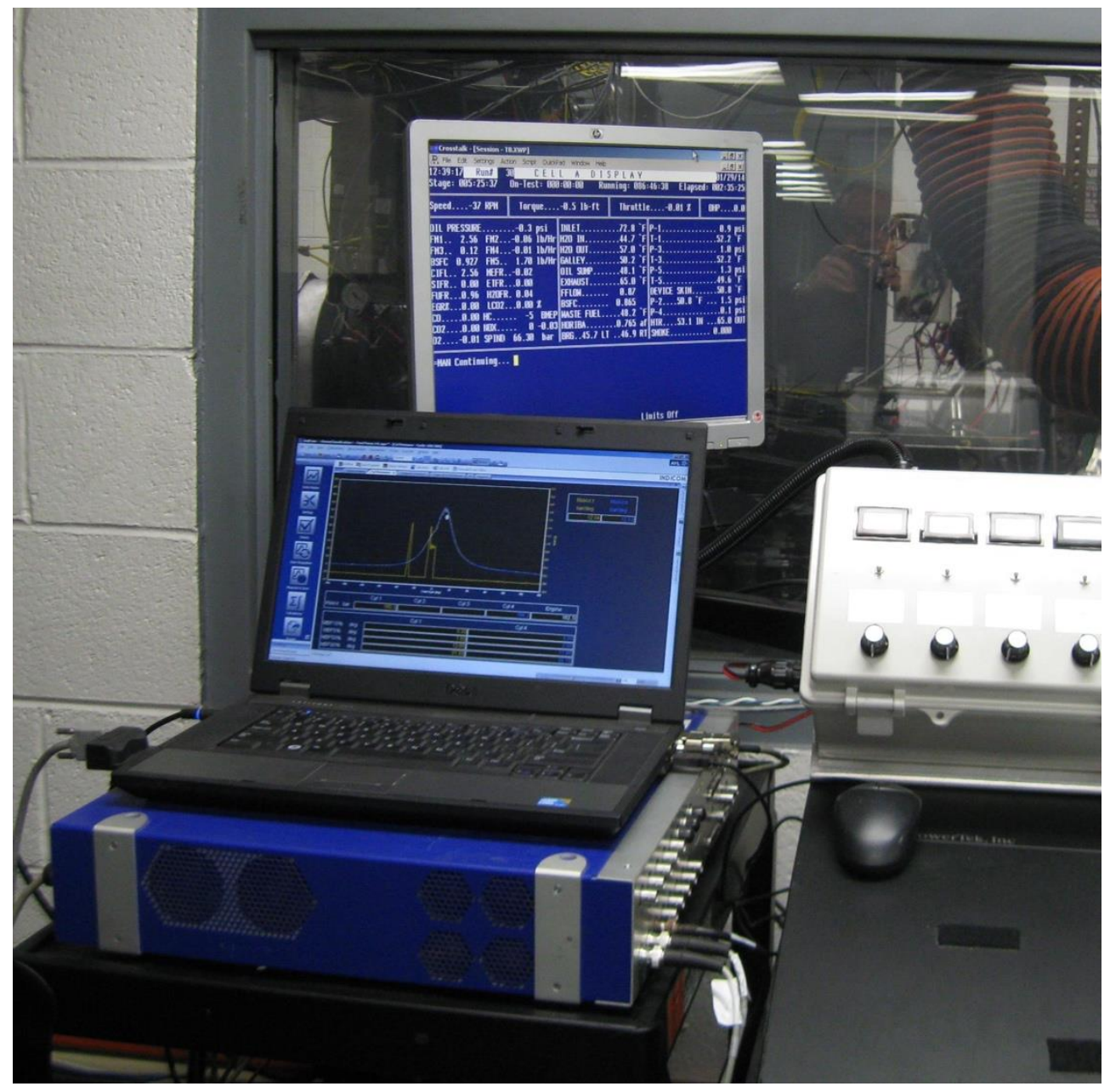

Figure 7. An example of a control system for monitoring the level of toxicity of exhaust gases of a diesel engine that uses diesel fuel of medium viscosity

In countries with a developed innovation economy, maximum attention is paid to the process of the correspondence of environmental standards to real conditions.

Therefore, when presenting, for example, on websites, information on the compliance of technical characteristics and capabilities of new products at the commercialization stages with all limitations of environmental standards, it also requires a preliminary analysis and, to some extent, a forecast of options for making these requirements and values still more stringent.

In the absence of direct options for comparing product indicators at the commercialization stage and tabular values of the restrictive requirements of relevant 
environmental standards, it makes sense to conduct a system search in specialized laboratories of institutes involved in the development of relevant standards and guidance materials and instructions.

\section{Criteria for assessing levels of environmental safety}

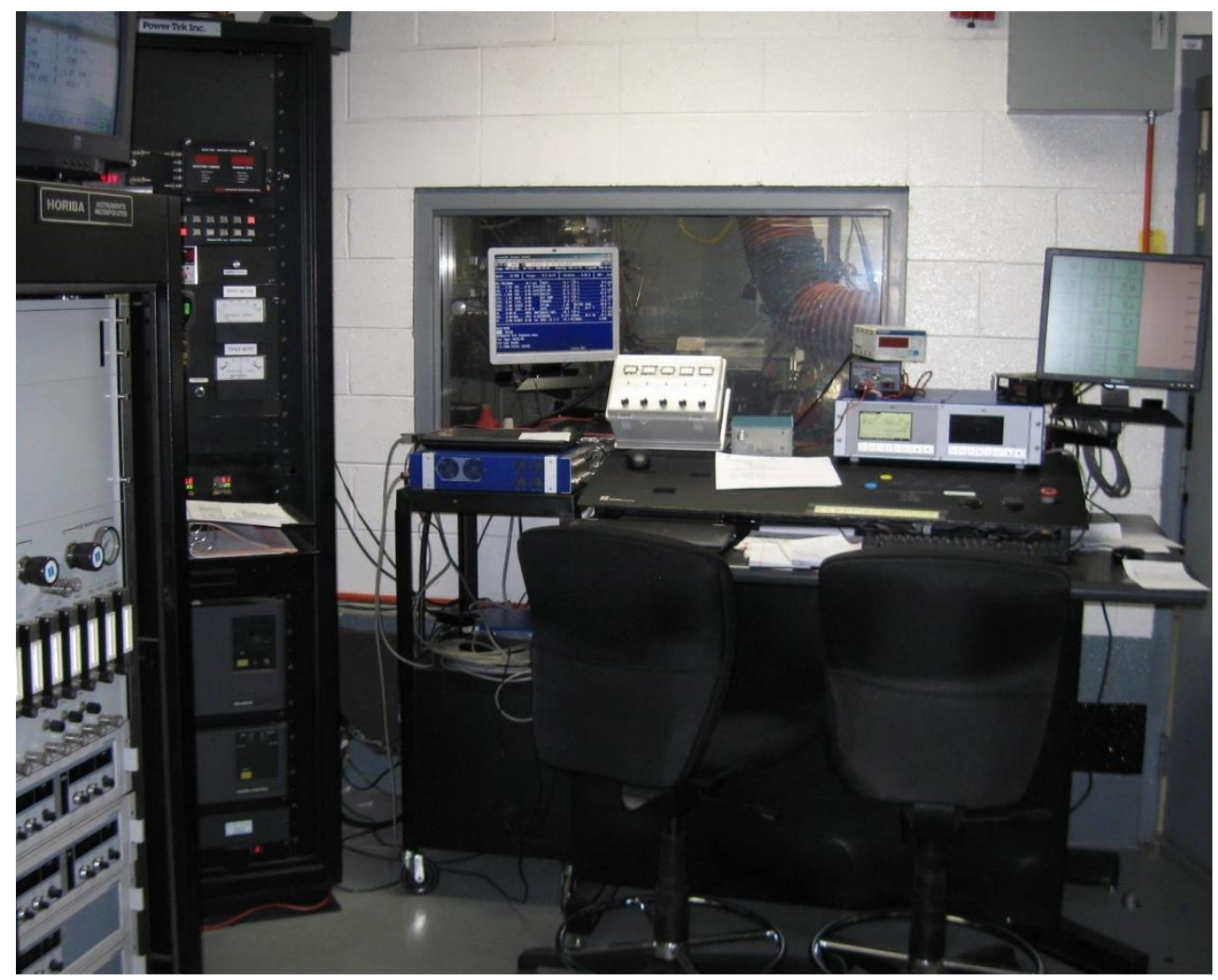

Figure 8. An example of a laboratory complex for analytical verification of compliance of the exhaust gases toxicity level with the requirements and limits of the current environmental standards

As for many product and product groups, indicators of their environmental characteristics are also complex and require, for example, the monitoring of the exhausts of internal combustion engines, and simultaneous monitoring of thousands of parameters. Systematic assessment and comparison of the reference level of environmental safety with actual indicators of a product or item, requires specially developed tables of comparison 
with mathematical models of analytical and comparative evaluation of the same kind of environmental safety to be included in the relevant, official documents.

\section{References:}

1. US patent application No20180290403, 11.10.2018. Unitized Composite Structure Manufacturing System / Hasan; Zeaid F.; et al.

2. Patent Application USA No20180285065, 04.10.2018. SMART CONTROLLING DEVICE AND METHOD OF CONTROLLING THEREFOR / JEONG; Gyuhyeok

3. US patent application No20180293778, 11.10.2018. SMART COMPRESSION/DECOMPRESSION SCHEMES FOR EFFICIENCY AND SUPERIOR RESULTS / Appu; Abhishek R.; et al.

4. US patent application No20180285306, 04.10.2018. INTERNET PROTOCOL (IP)-ENABLED SMART TRANSDUCER / ESSMANN; ROLAND; et al.

5. US patent application No20180272023, 27.09.2018. SMART OPTIC CONTROLLER FOR A HYDROXYL GENERATOR UNIT / BYSTRZYNSKI; Richard Mariusz; et al.

6. US patent application No20180272657, 27.09.2018. DISPLAY PART PROTECTOR FOR A SMART DEVICE AND METHOD OF ADHERING THE DISPLAY PART PROTECTOR TO THE SURFACE USING THE DEVICE / RYU; JONGYUN; et al.

7. US patent application No20180270076, 20.09.2018. SMART NETWORKING OF TRADITIONAL APPLIANCES / Natarajan; Sreekanth; et al.

8. US patent application No20180270799, 20.09.2018. METHOD AND APPARATUS FOR DOWNLINK CONTROL INFORMATION DESIGN FOR NETWORK COORDINATION / NOH; Hoondong; et al. 
9. US patent application No20180293366, 11.10.2018. SYSTEMS AND METHOD FOR SECURELY SHARING AND EXECUTING DATA AND MODELS /

Subramaniyan; Arun Karthi; et al.

10. US patent application No20180299849, 18.10.2018. SYSTEMS AND METHODS OF HIERARCHICAL SMART ASSET CONTROL APPLICATION DEVELOPMENT AND OPTIMIZATION / MARTIN; Peter G; et al.

Appendix 1

United States Patent Application 20180290403 Kind Code

Unitized Composite Structure Manufacturing System

\begin{abstract}
A method and apparatus for a composite structure manufacturing system. The system comprises a heating system and one or more smart bladders that encompass the heating system. The heating system and the one or more smart bladders form a curing tool defining a volume for the composite structure when one or more smart bladders in the one or more smart bladders is in a rigid state.
\end{abstract}

Appendix 2

United States Patent Application 20180285065

Kind Code 


\begin{abstract}
The present specification relates to a smart controlling device capable of utilizing machine learning for voice recognition and a method of controlling therefor. The smart controlling device according to the present invention includes a receiver configured to receive an input including a command trigger, and a controller configured to detect one or more external display devices, select a display device of the detected one or more external display devices, cause a power status of the selected display device to be changed to a first state, and cause a response data corresponding to a first command data received after the command trigger to be output on a display of the selected display device.
\end{abstract}

Appendix 3

United States Patent Application 20180293778 Kind Code

SMART COMPRESSION/DECOMPRESSION SCHEMES FOR EFFICIENCY AND SUPERIOR RESULTS

\begin{abstract}
A mechanism is described for facilitating smart compression/decompression schemes at computing devices. A method of embodiments, as described herein, includes unifying a first compression scheme relating to three-dimensional (3D) content and a second compression scheme relating to media content into a unified compression scheme to perform compression of one or more of the 3D content and the media content relating to a processor including a graphics processor.
\end{abstract}


Appendix 4

United States Patent Application

20180285306

Kind Code

A1

ESSMANN; ROLAND; et al.

October 4, 2018

INTERNET PROTOCOL (IP)-ENABLED SMART TRANSDUCER

\begin{abstract}
An Internet Protocol (IP)-enabled smart transducer includes a sensor for generating field data regarding a physical quantity associated with processing equipment or a device in an industrial processing facility, and a signal conditioning circuit for at least one of amplifying and filtering the field data to provide conditioned field data. A communications interface is coupled to the signal conditioning circuit including a processor having an associated memory and a field data to IP data conversion algorithm for generating the IP data from the conditioned field data, and a transmitter is for transmitting the IP data across an IP bus to at least one application connected to the IP bus.
\end{abstract}

Appendix 5

United States Patent Application

20180272023

Kind Code

A1

BYSTRZYNSKI; Richard Mariusz; et al.

September 27, 2018

SMART OPTIC CONTROLLER FOR A HYDROXYL GENERATOR UNIT

Abstract 
A hydroxyl generator unit includes an ultraviolet light source, a reaction chamber within interior space of the hydroxyl generator unit, environmental sensors and a smart optic controller coupled to environmental sensors and to the ultraviolet light source. The smart optic controller integrates environmental conditions, and in response to at least the environmental conditions, the smart optic controller generates an output signal to the ultraviolet light source to control to the hydroxyls generated by the hydroxyl generator unit. The smart optic controller includes at least one of an air flow sensor, a temperature sensor, a humidity sensor and a light sensor, to control operation of the hydroxyl generator unit. The smart optic controller includes a microcontroller that interrogates the sensors and that interfaces to external systems. The smart optic controller includes a near field communications circuit attached to a sensor communications link, and a control interface via an RS-485 connection.

Appendix 6

United States Patent Application

20180272657

Kind Code

A1

RYU; JONGYUN; et al.

September 27, 2018

DISPLAY PART PROTECTOR FOR A SMART DEVICE AND METHOD OF

ADHERING THE DISPLAY PART PROTECTOR TO THE SURFACE USING THE DEVICE

\section{Abstract}

Disclosed are a protector having a protecting and restoring function of a display part of a smart device and a method of adhering the display part protector. The display part protector for the smart device according to the present disclosure includes a protector part adhered to at least one of a flat display area, and a curved display area of a smart device having at least one of the flat display area and curved display area and an adhesive layer 
which is formed by spreading a flow-able adhesive composition between an entire area of a lower surface of the protector part and a display area of the smart device and then curing the flowable adhesive composition and adheres the entire area of the lower surface of the protector part to the flat display area and the curved display area.

Appendix 7

United States Patent Application

20180270076

Kind Code

A1

Natarajan; Sreekanth; et al.

September 20, 2018

\title{
SMART NETWORKING OF TRADITIONAL APPLIANCES
}

\begin{abstract}
This disclosure provides systems, methods and apparatus, including computer programs encoded on computer storage media, for an appliance interface apparatus. In some implementations, the appliance interface apparatus enables network-based control of a traditional appliance. The appliance interface apparatus includes an appliance socket to connect the traditional appliance to a power source. The appliance interface apparatus includes a first network interface for establishing a first communication link with a smart appliance controller in a network. The appliance interface apparatus can establish a second communication link with a control circuit for controlling the traditional appliance. Upon receiving a command from the smart appliance controller, the appliance interface apparatus sends a signal via the second communication link to the control circuit to control an operation of the traditional appliance in accordance with the command.
\end{abstract}

Appendix 8 


\title{
METHOD AND APPARATUS FOR DOWNLINK CONTROL INFORMATION DESIGN FOR NETWORK COORDINATION
}

\begin{abstract}
A method and apparatus for downlink control information (DCI) design for network coordination (coordinated multi-point transmission (CoMP)) are provided. In addition, a method and apparatus for configuration of demodulation reference signal (DMRS) and transmission and reception of DMRS, and a method and apparatus for transmitting and receiving uplink signal according to uplink transmission scheme are provided. The disclosure relates to a communication method and system for converging a 5th-generation (5G) communication system for supporting higher data rates beyond a 4th-generation (4G) system with a technology for internet of things (IoT). The disclosure may be applied to intelligent services based on the 5G communication technology and the IoT-related technology, such as smart home, smart building, smart city, smart car, connected car, health care, digital education, smart retail, security and safety services.
\end{abstract}

Appendix 9

United States Patent Application

20180293366

Kind Code

Subramaniyan; Arun Karthi; et al.

October 11, 2018

SYSTEMS AND METHOD FOR SECURELY SHARING AND EXECUTING DATA AND MODELS

\section{Abstract}


A simulation computer device for securely executing a model includes at least one processor in communication with at least one memory device. The simulation computer device is configured to store a smart container including a model and a usage policy. The simulation computer device is also configured to receive a plurality of inputs for the model and determine whether to validate the model based on the usage policy. The simulation computer device is further configured to execute the model with the plurality of inputs if the model was validated. Moreover, the simulation computer device is configured to transmit at least one output.

Appendix 10

United States Patent Application

20180299849

Kind Code

MARTIN; Peter G; et al.

October 18, 2018

SYSTEMS AND METHODS OF HIERARCHICAL SMART ASSET CONTROL APPLICATION DEVELOPMENT AND OPTIMIZATION

\begin{abstract}
Systems and methods of a Hierarchical Smart Asset Control Application development and Integrated Smart Asset Control System optimization are disclosed. In various embodiments, the system may develop a Hierarchical Asset Control Application and corresponding control hardware requirements. This can be used to create an Integrated Smart Asset Control System in order execute various processes for a set of equipment elements. The smart assets associated with the system may utilize intelligent agents to balance operational constraints and operational objects in order to determine real-time optimized operational parameters for a process and implement the appropriate controls to facilitate achieving the improved operational objectives.
\end{abstract}

